\title{
Building a Pillowfort: Political Tensions in Platform Design and Policy
}

\author{
BRIANNA DYM*, University of Colorado Boulder, USA \\ NAMITA PASUPULETI ${ }^{*}$, University of Colorado Boulder, USA \\ CASEY FIESLER, University of Colorado Boulder, USA
}

Social media platforms make trade-offs in their design and policy decisions to attract users and stand out from other platforms. These decisions are influenced by a number of considerations, e.g. what kinds of content moderation to deploy or what kinds of resources a platform has access to. Their choices play into broader political tensions; social media platforms are situated within a social context that frames their impact, and they can have politics through their design that enforce power structures and serve existing authorities. We turn to Pillowfort, a small social media platform, to examine these political tensions as a case study. Using a discourse analysis, we examine public discussion posts between staff and users as they negotiate the site's development over a period of two years. Our findings illustrate the tensions in navigating the politics that users bring with them from previous platforms, the difficulty of building a site's unique identity and encouraging commitment, and examples of how design decisions can both foster and break trust with users. Drawing from these findings, we discuss how the success and failure of new social media platforms are impacted by political influences on design and policy decisions.

CCS Concepts: • Human-centered computing $\rightarrow$ Empirical studies in collaborative and social computing; Computer supported cooperative work; Social media.

Additional Key Words and Phrases: online communities, platform design, policy, social media, fandom

ACM Reference Format:

Brianna Dym, Namita Pasupuleti, and Casey Fiesler. 2022. Building a Pillowfort: Political Tensions in Platform Design and Policy. Proc. ACM Hum.-Comput. Interact. 6, GROUP, Article 16 (January 2022), 23 pages. https: //doi.org/10.1145/3492835

\section{INTRODUCTION}

Whenever a social media platform is mired in controversy, a common rallying cry emerges: "let's go somewhere else." Concerns over privacy, for example, can drive people to leave Facebook [57]. Sometimes people can successfully move elsewhere, like fan communities did when they left LiveJournal for Tumblr and Archive of Our Own [22]. Often, threats to leave a social media platform are empty. For example, when Reddit banned several subreddits in 2015, people moved into other subreddits and joined the Reddit clone Voat, but Reddit did not suffer an extreme loss of user activity despite some people claiming that the ban signaled the end of Reddit [13].

${ }^{*}$ Both authors contributed equally to this research.

Authors' addresses: Brianna Dym, brianna.dym@colorado.edu, University of Colorado Boulder, Boulder, Colorado, USA; Namita Pasupuleti, namita.pasupuleti@colorado.edu, University of Colorado Boulder, Boulder, Colorado, USA; Casey Fiesler, University of Colorado Boulder, Boulder, Colorado, USA.

Permission to make digital or hard copies of all or part of this work for personal or classroom use is granted without fee provided that copies are not made or distributed for profit or commercial advantage and that copies bear this notice and the full citation on the first page. Copyrights for components of this work owned by others than the author(s) must be honored Abstracting with credit is permitted. To copy otherwise, or republish, to post on servers or to redistribute to lists, requires prior specific permission and/or a fee. Request permissions from permissions@acm.org.

(c) 2022 Copyright held by the owner/author(s). Publication rights licensed to ACM.

2573-0142/2022/1-ART16 \$15.00

https://doi.org/10.1145/3492835

Proc. ACM Hum.-Comput. Interact., Vol. 6, No. GROUP, Article 16. Publication date: January 2022. 
Large social media platforms like Facebook, Twitter, and YouTube are able to leverage massive resources to ensure that leaving their platforms is not feasible for most users [44]. Smaller platforms can carve out a niche among certain online communities, but also must entice users to join by providing certain features or policies to which major platforms won't commit. For example, the United States' 2020 election prompted major exoduses from Twitter and Facebook when the companies banned then U.S President Donald Trump from using their platforms. Users flocked to alternative platforms like Parler, Gab, and even MeWe, stating that the political interests of Facebook and Twitter no longer aligned with their values [37]. Value tensions can be a strong motivator in choosing to leave or join a platform [22]. In considering how those value tensions influence and alter social media platforms, we can examine how those platforms project their values through design and policy decisions and how users react to and potentially influence those values.

In this study, we observe values being negotiated between users and a new social media platform, Pillowfort, but we also see how those values push against and are shaped by a broader set of politics that can ultimately eclipse those values. It is those political forces (e.g. federal regulations shaping a platform's policies) that we bring into focus through our analysis.

Values, in the context of this research, can be understood as individual priorities, desires, internalized rules, and beliefs that a person or group of people hold. Values can be leveraged for political purposes to appeal to certain projects, rules, or forms of governance. But what are politics, more specifically? The definition of "politics" is slippery, but can broadly be understood as an agenda that prioritizes certain values over others, and seeks to enact those values through practices that prompt social change, maintain a status quo, or accumulate power, depending on what values are currently dominant. We draw this definition from Chantal Mouffe's discussion of political systems [51] and Ezio Manzini's framing of life projects as a means of transformative political power [46].

Social media platforms have politics in that they are situated within a social context that frames a technology's impact, but also have politics through their design that enforces certain power structures and serves certain authorities [61]. In the context of this research, we are interested in understanding politics as a means of exercising power, agency, and authority, especially from the perspective of how technologies might exercise those qualities [61]. Specifically, we are interested in how social media platforms relate to a broader political framework. We turn to politics as a means of understanding values in social media platforms for two important reasons.

First, social media platforms operate within political institutions. Huybrechts and colleagues introduce the concept of "institutioning" as a means of refocusing participatory design and codesign politics from a micro level to also consider meso- and macro-level political forces, thus challenging existing institutions [36]. In our own work, we leverage this shift in focus to highlight how certain technical design practices and values are controlled and limited by broader political institutions. For example, policy is just as influential in a technology's development arc as is design and practice [38]. Recently, social media platforms and other technology developers had to change their data collection practices to meet the General Data Protection Regulations (GDPR) standards for European Union citizens, thus prompting companies to significantly alter technical designs as well as their practices in working with user data moving forward. The GDPR projects a value of privacy and user rights over their data. However, the regulations themselves and their impacts are part of a political institution's policies surrounding privacy, data, and a person's rights.

Second, platforms not only are enacted upon by politics, they also collide with, influence, and serve broader political interests. Social media platforms can limit what sort of content is viewable depending on what country that content is being viewed in and that country's laws surrounding certain content. Platforms can also restrict what sort of content can be hosted on their platforms in response to laws that affect them. For example, in response to an Australian law that would require 
Facebook to pay news providers for their content, the platform restricted Australian users from sharing news on the platform altogether [28].

How social media platforms interact with broader political forces is increasingly important to a person's decision of whether or not to join a social media platform, and they can greatly impact when people choose to leave. Furthermore, there are increasingly stubborn barriers to a social media platform's success in the shape of broader political forces. As a case study, we examine Pillowfort to understand how new social media platforms encourage people to join. We also examine what struggles new social media platforms face in an increasingly competitive and monopolized internet landscape [44]. This case study highlights the design and policy choices that Pillowfort's developers make over a two-year period, how their users respond to them, and how politics, both in terms of a platform's policy and design features as they relate to larger political institutions, are surfaced throughout value negotiations between staff and users. In turn, this research highlights different barriers that new social media platforms face in encouraging newcomers to join and in building a sustainable platform.

We first approached Pillowfort as an example of a small social media platform being built by and for people within a specific online community. We asked the question, "What are the different barriers and opportunities involved in people building their own online spaces?" We asked this question in hopes of better understanding how to promote communities leveraging technology for empowerment. From our analysis of discussion posts between staff and users, we present findings that demonstrate (1) how Pillowfort makes design and policy choices heavily influenced by past experiences users have on other platforms; (2) how Pillowfort as a platform constructs its identity, how users perceive that identity, and how Pillowfort leverages that identity to encourage users to not only join, but stay; and (3) how Pillowfort developers both foster and break trust with users through specific choices in the design process. From those findings, we discuss how politics strongly influence the options people have in migrating between platforms, the design choices that those platforms can make, and how broader political forces can contribute to the success and failure of social media platforms.

\section{BACKGROUND}

As a case study, Pillowfort offers insight into how new social media platforms draw users in and away from more established competitors. Furthermore, Pillowfort is a small, independent social media platform that positions itself as fundamentally different from major social media platforms like Tumblr, Twitter, and Facebook. Pillowfort makes specific, political choices that draw in users. But, these choices also come with their own trade-offs. Pillowfort also contends with serious struggles that may or may not be surmountable for new social media platforms. In this section, we first provide a brief history of transformative fandom, the target community that Pillowfort sought to design itself for. We then ground this work in research on how people participate in and contribute to online communities, when online communities succeed and fail, and how social media platforms and their communities intersect with politics.

\subsection{Pillowfort and Participatory Culture}

We examine Pillowfort because of the staff's fairly transparent engagement with users during the "closed beta" of the site's development ("closed beta" meaning users need an account to create accounts and posts; the site itself is visible to the public). Staff regularly made posts announcing proposed changes to the site and openly engaged with user comments in discussion threads. The open discussion between staff and users forms a rich site of study for observing requirementsgathering processes for Pillowfort's ongoing development that would otherwise happen away from the public eye. 
Pillowfort opened itself to new members in August of 2018 following a successful Kickstarter campaign. The campaign page described Pillowfort as, "a new blogging platform that aims to improve the current state of social media by providing better privacy and communication tools." In its Twitter bio, Pillowfort describes itself as a "user-funded" blogging platform for fans and creators Though not directly stated, Pillowfort's design positions itself in direct opposition to Tumblr, which in 2018 was a major social media site for fan communities [22]. In December of 2018, Tumblr banned adult content from the site, thus driving many LGBTQ+ users and fan communities from the platform in fear that their content and communities would no longer be safe on the platform $[2,34]$.

Over the years, Pillowfort has steadily grown in size, starting from just over 10,000 according to their Kickstarter page to over 100,000 (according to their May 2021 community update). The staff regularly engages with their users (referred to by the staff as "Pillowfolks"), posting feedback surveys and talking to users in public discussion posts, eliciting values from the community to consider in design changes [30]. These posts span over two years of development and form a rich corpus of conversations about changes to Pillowfort's design and policies in that timeframe. In January of 2021, Pillowfort attempted to launch into an open beta, but several critical security issues caused the site to be taken offline for several months. In May of 2021, Pillowfort came back online in a resumed closed beta.

Pillowfort's lead architect and many of its developers come from the broader online community of transformative fandom (also known as "fandom"). As a result, Pillowfort is designed with the needs of fan communities in mind. This community primarily produces creative works derivative of original content. For example, someone might draw fan art of Disney princesses in modern clothes or write fanfiction about the off-screen adventures of Captain Kirk and Mr. Spock. While many sub-communities in fandom form around specific books or television shows, they all belong to a broader community identity that shares social norms and expectations for behavior [59]. Fan community members share strong social norms around copyright and attribution [21] as well as providing positive feedback toward one another's fanworks [12]. Fandom has historically and to this day served as a safe space for marginalized communities [27, 39]. For example, LGBTQ+ people use fan communities to explore their identity safely, away from harms they might experience for exploring that identity elsewhere, and to connect with LGBTQ+ communities and resources they cannot access anywhere else [18]. As a result, fan communities also have strong social norms around privacy and secrecy to keep those vulnerable community members safe $[11,20]$.

Transformative fandom operates as a type of participatory culture, meaning that people are engaged in a specific type of cultural production, like creating fanworks [40]. Fanworks are not the only sort of cultural production that fandom takes part in. The community is also deeply involved in the design and development of its own online spaces. For example, Archive of Our Own (AO3) is a collaboratively constructed site that was built by its own community's members, mostly women, many of whom learned to code through legitimate peripheral participation [23, 24]. AO3's construction is an example of infrastructuring in participatory design, wherein a community is generating critical infrastructures to support its own activities and needs [42]. The site's search infrastructure is maintained to this day by a team of tag wranglers, volunteers from the community who work on the back end of the site to pair user-generated tags to parent tags [10]. Participation is important to fan communities, not only for their continued existence, but also for their success.

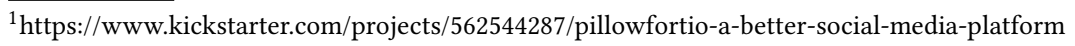




\subsection{Success and Failure in Online Communities}

Successful online communities usually require a critical mass of people joining that community $[14,41]$. Gaining and keeping that critical mass can be challenging, seeing as many social media platforms fail in some way, whether by being bought out and dissolved by a larger company, losing enough of its users to no longer be sustainable, or even by policy and regulatory changes that limit the platform's functionality. When considering Pillowfort as a case study, we want to examine how different social media platforms have come and gone, or stayed indefinitely, and what are the contributing factors to a platform's success or failure. Pillowfort, still in its beta phase, has encountered many barriers to success in its development process but has not failed yet, as we will discuss in our findings. The barriers that have impeded Pillowfort's success prompt us to consider how and why social media platforms might fail.

In 1988, Jonathan Grudin drew attention to the problem of why certain computer-supported cooperative work applications fail, outlining three problems that CSCW applications encounter. Grudin identifies one problem as the "disparity between who does the work and who gets the benefit" (p. 86 [33]). As an example, Grudin describes an electronic calendar application dependent on everyone participating in updating their own electronic calendar within a company's system. If they do not participate, then the calendar's scheduling function will not work. There is also the problem of the social-technical gap in that the social desires people have for a CSCW application are not always technically feasible [1].

Group work is messy, and there are often more avenues to failure when an application relies on groups. As a social media platform, Pillowfort needs to not only reach a critical mass of users [53], it also needs to provide technical features desirable and feasible to maintain [1,33]. Chang and Yang note that critical mass for adopting new social media platforms (in their example, blogs) requires both participation and collective actions from all individuals who are affected by the technology (p. 374, [14]). In other words, people must commit to using the application and interacting with other users in a way that creates meaningful social interaction. Social media platforms dependent on user-generated content struggle with this challenge. On Wikipedia, for example, people must transition from being readers to actively contributing to articles if the site is to maintain quality and diverse information [9].

Active participation is not the only kind of participation needed to keep a social media site thriving. Passive or less visible participants like readers on Wikipedia, for example, are an important part of the community. Readers indicate an article's engagement, or even its perceived reliability as a source of information [5], important data for the people who maintain Wikipedia articles. In order to be a successful online community, platforms need to draw in users that commit to joining and participating in using the platform.

Increasingly, users make decisions to join or leave social media platforms based on a platform's regulations and rules. Policy plays a significant factor in a social media platform's success or failure. What a social media platform's policies allow and restrict as well as how policies are enforced greatly impacts users. In the case of fan communities especially, policy changes can drive a significant amount of traffic to and from different social media platforms [22]. How those policies are formed and how they inform user interactions are all strongly tied to political considerations-both in how a social media platform engages with politics and how political forces might shape a social media platform.

\subsection{Politics, Values, and Social Media Platforms}

Technologies interact with politics in a variety of ways. For example, we can leverage technical design to promote widespread engagement with political frameworks like environmental justice 
[17]. Bitcoin is purposefully stylized as a decentralized autonomous platform that is free from politics, but does in fact consolidate its power into a relatively small group of people and perpetuate inequalities [45]. Google's search engine reflects certain biases back at its users that enforce different stereotypes and prejudices like racist and sexist beliefs [52]. Optimization features on Facebook's ads lead to gender and racial biases in who those ads are served to, reducing opportunities for women and people of color [3]. Bias and discriminatory practices in social computing play into politics in that they, whether intended or not, serve to uphold power structures that largely benefit people already in positions of authority. In our own work, we examine how values elicited throughout Pillowfort's design process come into conflict with broader political forces. In our analysis and discussion, we center those politics to better understand their impact on and control over designing social media platforms. Here, we discuss a specific example of values conflicting with politics on social media. We then review prior work around values, politics, and design.

2.3.1 Values versus Politics in Social Media. The social media platform Reddit previously created a "toxic technoculture" through policy and design choices that prioritize a specific political enterprise, empowering some and disenfranchising others [48]. Massanari uses the example of "the fappening" to illustrate how Reddit promoted a toxic environment for monetary gain. The "fappening" was an event where Reddit users uploaded leaked nude photographs of women celebrities to share across the internet. Despite the photographs being illegally obtained, Reddit staff did not initially honor requests to remove or ban the content from being shared. The subreddit the photographs were shared to generated a significant amount of money and traffic for the site, Massanari notes [48]. It was not until some of the photographed subjects were identified as minors (under 18) that Reddit staff removed the content. In this example, Reddit takes a stance that devalues the women shared in those photographs (prioritizing monetary incentives) until regulatory laws prohibiting hosting explicit imagery of minors requires the photographs be removed. Reddit's values (prioritizing the monetization of a popular subreddit) were inevitably altered by the regulatory power of the U.S. government.

Reddit's values are not necessarily a static monolith, and there are ways that typically marginalized groups have found important outlets for their voices on the site [49]. Even so, maintaining certain community standards on Reddit is challenging in the face of the site's underlying expectations of acceptable behavior. Reddit's administrators in the past have attempted to alter what sort of content is seen as appropriate through banning subreddits dedicated to hate speech [13]. However, many of Reddit's users still push back against subreddit rules and moderation decisions that devalue sexist and racist perspectives [32].

2.3.2 Values \& Design. Reddit's developers face several challenges in platform governance, and though the social media site is not part of the broader monopoly of tech companies like Facebook, Google, and Amazon, it still has significant resources it can levy in addressing challenges where values come into conflict with politics. Values, whether held by users or developers, operate in a larger political framework that platform developers must operate within to some degree. In this sense, values are situated as a subset within political institutions.

Design practices like participatory design [55], value-sensitive design [30], and "values in design" [26] center humans and their personal values in the technology design process. Though all three methods have different strategies for accomplishing this task, they share the common goal of centering ethical and democratic design and use of technology [7]. For example, Bassetti and colleagues use a co-design project to explore alternative social media platform design that counteracts the "commodification of digitally-mediated cooperation" (p. 257, [7]). The resulting platform, Commonfare, embodies specific anti-capitalist values in its design that in turn push back against the political institution of "platform capitalism" [56]. 
Designing for a community's values is not necessarily an easy process. Online communities often have competing values, or value tensions that designers must negotiate [50]. For example, while certain fan communities share strong overarching norms and values [23], those values still differ at critical points, resulting in value tensions that must be negotiated through design [20]. Certain value tensions can be construed as "value dams," e.g. a strongly held value that only a small number of community members possess, yet nonetheless will still limit overall appropriation of a technology [50]. Meanwhile, commonly held values can function as "value flows," encouraging broad technology adoption from the community when the appropriate value is present [50]. In our analysis of Pillowfort, we examine how the site's developers appeal to strongly held community values, but are often thwarted or limited by strong political barriers related to legal issues, funding, and resource availability. In the following section, we review politics and political ideology more broadly and how it intersects with social media technologies.

2.3.3 Politics \& Design. Inevitably, online communities express values that influence that community's design. However, those communities are also subject to powerful and controlling political forces. In contending with those forces, institutioning is offered as a means of transforming larger political structures through participatory design processes [36]. Participatory design, much like participatory culture, is positioned as a means of democratizing commodified technical systems $[36,40]$. Historically, the design practice is leveraged to shift power into the hands of workers directly interacting with a technology rather than consolidating power in the hands of a few specialists [55].

While this design practice is certainly important in affecting change, there is the problem that not all politics can be changed by technical design, and not all designers are empowered to push against certain political structures. Smaller social media platforms like Pillowfort are confronted with almost insurmountable challenges in navigating the digital landscape. In The Gentrification of the Internet, Jessa Lingel argues that the modern internet is currently gentrified, with digital real estate consolidated to a few companies. It is through displacement, isolation, and commercialization that online communities are splintered and reshaped to fit the expected communal spaces that major tech companies design, thus enforcing a specific set of politics through design [44]. Smaller and newer social media platforms must also consider these politics in their own design. The expectations a user might have toward a social media platform's design may not always be feasible to meet, especially for small, resource-limited platforms.

Despite these challenges, small platforms and their communities have opportunities to confront politics in meaningful ways. Through building the Commonfare site, for example, Bassetti and colleagues found that designing for common values promoted autonomy and an alternative to commodified social media platforms [7]. In our analysis of staff conversations with Pillowfort users, we highlight themes of how politics intersect with the platform's design and policy in addition to how those intersecting challenges influence a user's commitment to Pillowfort. In the following section, we describe our methods of data collection and analysis for compiling these findings.

\section{METHODS}

Our research team began data collection by compiling a list of all public posts made by the official staff account for Pillowfort staff members. This list began with the staff's first public post on August 4, 2018 to when we stopped data collection in June of 2020. We recorded the post's title and URL, the topic of the post, how many replies it had, and whether or not it was a new post made by staff or a reblog (for example, the staff reblogged links to surveys multiple times). We then coded each staff post to determine if it was discussing any of the following:

(1) Whether or not it was announcing a change to the site 
(2) Whether or not it was collecting feedback on possible changes to be made to the site

(3) Whether or not it was discussing policy changes

Any posts that met at least one of these criteria that also had at least 50 public comments were marked and then recorded, along with all publicly available comments, into a document for further analysis. This selection process resulted in collecting 12 separate documents of an entire discussion post and their associated comments. The largest document contained 50,879 words and the smallest document contained 1,319 words, with the total corpus word count being 130,016 words. We analyzed 12 original posts from the staff account and 1,839 total comments.

There are two outliers to this criteria: (1) an early site update that received 76 comments was not publicly available at the time of data collection and so was not included in analysis; and (2) an announcement on the Black Lives Matter protests in the summer of 2020 only received 20 comments, but due to the announcement's focus on a major historical event researchers determined that it was important to analyze along with the rest of the corpus. Table 1 on the next page displays descriptive data on the contents of each staff post.

Table 1. Pillowfort Discussion Posts

Topic Site Change Feedback Policy Word Count Total Comments

\begin{tabular}{|clllcc}
\hline Combating Racism & No & Yes & No & 1551 & 20 \\
\hline Community Survey 1 & No & Yes & No & 7059 & 162 \\
\hline Community Survey 2 & No & Yes & No & 1319 & 50 \\
\hline Community Survey 3 & No & Yes & No & 3859 & 116 \\
\hline Community Update & Yes & No & No & 11,170 & 132 \\
\hline Site Change 1 & Yes & No & No & 5508 & 61 \\
\hline Site Issues & No & Yes & No & 29,035 & 450 \\
\hline Account Changes & Yes & No & Yes & 9634 & 126 \\
\hline Policy Change & Yes & No & Yes & 50,879 & 522 \\
\hline Site Update 1 & Yes & No & No & 3544 & 82 \\
\hline Site Update 2 & Yes & No & No & 4339 & 66 \\
\hline Site Update 3 & Yes & No & No & 2119 & 52 \\
\hline
\end{tabular}

This selection criteria prioritizes staff posts that contain extensive discussion among users about how Pillowfort is growing, implementing new changes and decisions, and negotiating potential new changes to the site. Each post unfolded as a conversation involving staff and users, with users sometimes speaking directly to staff and sometimes speaking directly to one another. These conversations tie into a broader discourse about Pillowfort as a whole.

The first two authors conducted a discourse analysis on all posts and their comments. The authors paid particular attention to what the staff post elicited from its audience and how staff responded to user questions in the comments themselves, in addition to how users addressed one another. For example, staff frequently posted community feedback surveys where users could submit targeted feedback through a survey form, but would also submit questions, comments, and ideas for site development to the accompanying discussion thread. A survey's topic often informed what people would continue to talk about in the discussion threads. When staff surveyed people about "community" (e.g. grouping) features, users frequently posted follow-up comments about current issues with or future hopes for "community" features on Pillowfort. However, users frequently drew 
upon shared experiences from other online fan spaces (like LiveJournal and Tumblr) to contextualize their comments and requests. When encountering references to prior events on other platforms, the authors met and discussed those events and how they affected current conversations.

Discourse analysis was well-suited for this corpus because we wished to observe how multiple conversations around Pillowfort's development formed over time and how those conversations related back to broader social concerns and historical contexts that could affect those conversations [31]. We also wished to better understand how those conversations fit into a broader social discourse about not only Pillowfort's development, but also longstanding hopes and anxieties around this particular community settling into new online spaces. We know from prior work that fan communities in particular (Pillowfort's primary audience) have a long and complex history around migrating between social media platforms [22].

The first two authors conducted their analysis by reading the staff posts and noting the major elements staff were communicating in their post. We then read through user replies, paying close attention to threaded discussions where users talked to each another and where staff replied to user comments and questions directly. In examining these conversations, we coded what users were responding to in staff posts as well as what outside subjects users were introducing to the conversation. We referred to Gee's discourse analysis toolkit to prompt questions we asked of the data [31]. For example, the "fill in tool" asks analysts to consider what context is missing from the conversation itself and to discover that context. Other prompts ask analysts to consider how identity is enacted and communities are constructed through the overarching discourse. We coded the data with these prompts in mind, marking where people made comparisons between Pillowfort and other spaces, how individuals sought to shape the tone of conversation, when and where staff chose to respond to user comments, and how all of those discursive acts tied back to the design trajectory of Pillowfort.

Throughout the analysis, the first two authors met weekly and discussed recurring themes they saw across multiple different staff posts. The authors began by consolidating similar codes into groups, writing a summary of those groups, and sharing those summaries and coded data with the third author. All of the authors then iteratively discussed and refined these groupings until we had sufficiently identified salient categories these groups fit into. Our goal in this process was to identify significant overarching trends throughout the two years of discourse over Pillowfort's construction. At the end of this process, we identified three major themes that dominated the discourse around Pillowfort's growth and evolution as a social media platform: (1) users compared Pillowfort to other platforms and expressed fears toward Pillowfort becoming like other platforms, or perhaps imagined how Pillowfort could better emulate existing platforms, introducing several design tensions that Pillowfort had to navigate throughout its development; (2) staff and users constantly discussed how changes to the site would impact Pillowfort's identity; (3) staff leveraged many different strategies to foster trust in their platform and users responded to indicate the level of trust they felt toward staff.

\subsection{Ethical Considerations}

Because our analysis draws on publicly available data that was not intended for research purposes, we have carefully considered the appropriateness of using Pillowfort as a site for analysis. Though people need an account to make posts, the site is publicly viewable and searchable by everyone unless a user has marked their content as private. For example, anyone can navigate to the staff's blog and view it whether or not they are logged in. Pillowfort is publicly viewable by design because, as we discuss in the findings, artists need to be able to link their posts on other websites and have them remain viewable to other audiences. Topics from our findings that might be considered 
potentially sensitive (e.g., around adult content or security issues) have been discussed publicly by the site's developers and covered in the media [60].

However, most social media users post content online without the expectation that their data will be observed by researchers [25]. Additionally, people in fan communities in particular have heightened concerns toward data privacy $[11,19]$. For example, closeted LGBTQ+ people in fandom worry that outsiders or family members might recognize their fandom profile from enough context clues or from recognizing their art style, though these concerns are largely due to the potential for amplification of content beyond its original context. [19]. With respect to reporting results from analysis of public data, even the small chance of harm can be mitigated by limiting the searchability and discoverability of content. Therefore, we present any quotes from both users or staff as paraphrased. That is, we rewrite each quote so that it is essentially the same sentiment originally expressed by a user or staff, ethically fabricating data representative of our observations [47]. We have given users unique identifiers to replace their usernames, and keep staff posts labeled as posted by staff. Moreover, research into the ethics of studying content from fandom revealed that fans tend to generally trust researchers, particularly if they are part of the community [19]; we note that the authors all have experience with transformative fandom and are familiar with the norms of the community.

In the following section, we explore our three key findings and highlight how they relate to Pillowfort's development as a social media platform.

\section{FINDINGS}

Our findings explore how user experiences on past social media platforms influence design and policy decisions on Pillowfort. We also examine how Pillowfort purposefully cultivates a certain identity as a social media site and how it mitigates threats to that identity. With user experiences on social media spanning decades, people arriving at Pillowfort have formed judgements toward the site based on features as benign as Pillowfort's color scheme. Finally, both the staff and users engaged in dialogue about trust, both building it and losing it with design and policy decisions. These findings point to complex choices and compromises both platform developers and their users make in committing to a social media platform-compromises that relate to a set of politics that influence Pillowfort's success and what it can ultimately be for its users.

\subsection{Design Tensions with Past Platforms}

Our first finding concerns how Pillowfort's developers addressed design challenges tied to perceived problems users identified when Pillowfort's design transgressed too closely to other platforms. People arriving at Pillowfort come with experiences and expectations shaped by previous platforms, especially large, well-established platforms. Newly arriving users expressed their hopes for what Pillowfort could be and how it would be able to serve their needs, as well as their fears of how Pillowfort might let them down like other platforms had.

Pillowfort is a relatively new site made with fan communities and their needs in mind. As a result, the people who came to Pillowfort compared it to other fandom-focused platforms like Tumblr, LiveJournal, and Archive of Our Own (and to a lesser extent, Twitter). Users had joined Pillowfort because social media platforms like Tumblr and Twitter had changes that users disagreed with or had fundamental objections to.

U1: I left Tumblr because it stopped letting you search individual blogs, and that's what I spent most of my time doing. It's a feature that I would love to see here!

User 1 wants Pillowfort to implement a search feature that Tumblr removed. Many users coming to Pillowfort were looking for a replacement to Tumblr. Users left Tumblr for Pillowfort most 
commonly because of the site's adult content ban, and any hint that Pillowfort might change their design or policy to negatively impact adult content was poorly received by users.

U2: Please don't hide not-safe-for-work content from non-users. Tumblr did this and it hurt traffic to the site so much even before the site-wide NSFW ban.

This user is responding to a community discussion about how not-safe-for-work (NSFW) content is filtered on Pillowfort. The developers had just restricted accounts belonging to users under 18 from viewing NSFW content. By doing so, users were concerned that adult content would not be viewable by anyone logged out of the platform, or that perhaps adult or "mature" content would be even more challenging to view in general. Historically, what counts as adult content within fandom tends to overwhelmingly include LGBTQ+ content of any variety, thus potentially limiting the resources and communities that LGBTQ+ groups have access to online [22]. In the case of Pillowfort, the developers have made no restrictions to viewing NSFW content while logged out, but the slight change still caused users to be worried that Pillowfort would turn into Tumblr, particularly in terms of blocking and flagging LGBTQ+ content for removal. Staff had to constantly design around this concern, even to the point of avoiding aesthetic changes that reminded people too much of Tumblr.

U3: I don't like that likes and reblogs add numbers to the notification sidebar. I was excited for some new conversation and it was just a bunch of passive likes and reblogs. That was honestly Tumblr's biggest failure as a social platform.

U4: This update does give Pillowfort that Tumblr blue color...I don't know, I haven't been on Tumblr in so long. But it does give me those vibes for one reason or another.

U5: I like the blue and purple. But I agree with what others are saying, that the blue makes Pillowfort look too much like Tumblr.

Even just a color similar Tumblr was enough to disappoint users. Pillowfort staff need to strike a careful balance between providing certain functions that users want imported from Tumblr while also being distinct from it. Users do not want a Tumblr clone, but they also want a refuge to flee from Tumblr.

Pillowfort is a small platform working with limited resources. The site's landing page looks similar to that of Tumblr or Twitter, and features rotating art from one of the site's users like Tumblr's home page does. The user is prompted to log-in with no obvious way to navigate to the site itself. However, like both Tumblr and Twitter, all users have the options to make their blogs public. Pillowfort goes a step further for privacy features as opposed to Tumblr and Twitter and allows for users to adjust the privacy setting on each post, which is a setting that users from fan communities value for safety reasons [20]. Artists in particular value this feature because it allows them to pick which posts are visible to the public and which are kept private for certain users or communities on Pillowfort. Before more elaborate privacy controls were added, users frequently requested them:

U6: I would really love more privacy features. What we can currently do is nice, but I really want the kind of privacy controls that LiveJournal had. I want to be able to group users and determine which groups can see which posts. This is a really important feature to me.

Currently, Pillowfort describes its "Content Control \& Privacy" as a major selling point for why people should join, with the log-in page featuring this description about how users can manage content: "Control how others interact with [all of your content] as well as how your post can be shared-and if other users can comment. Every post has the option to be visible to everyone, visible by your followers or visible only to yourself." The ability to have granular privacy controls is inspired by LiveJournal's original design, and has been a desired feature among fan communities for a long 
time [20]. Users described a sense of loss in privacy on other social media platforms and would occasionally get uncomfortable if Pillowfort adjusted its design in a way that users felt breached their privacy. For example, when the site started collecting date-of-birth information, some users felt it was a step in the wrong direction.

U7: Blogs are a place to share thoughts and feelings, sometimes intimate ones. If you're using Pillowfort to air grievances anonymously, the thought of providing identifiable information to the site is worrying. Especially now that internet vigilantism is popular thanks to certain parts of Twitter.

Here, we see a user comparing their concerns for providing identifiable information to Pillowfort with how users behave on other social media platforms. Despite Pillowfort and Twitter having extremely different designs (long-form blog posts and community discussion threads versus microblogging), users still fear that the "wrong" changes to the platform might send it careening in an undesirable direction. Pillowfort's developers are in the precarious position of dealing with a long checklist of things it cannot be (e.g. too blue or too invasive). Meanwhile, developers must also provide enough design features similar to and improved upon from other social media platforms to draw users away and make them commit to using Pillowfort. The developers must also accomplish all of this with less resources than many other social media platforms have access to.

\subsection{Building Pillowfort's Identity}

Despite needing to provide features similar to other platforms, Pillowfort draws in users by stylizing itself as different from major commercialized platforms. Scroll down on the site's front page, and Pillowfort advertises its claims to fame: content control and privacy, user-generated communities, blacklisting, blocking, threaded comments, and allowing users to post NSFW content (e.g. mature or "adult" content). Potential users can sign up through two different ways: through a registration key that has been distributed to them or by paying a one-time $\$ 5$ fee. At the bottom of the home page, Pillowfort explains why newcomers can pay a membership fee to join, "we chose to be a primarily user-funded platform without any financial assistance from investors or venture capital funding."

In addition to turning down external funding sources, Pillowfort also does not sell ads on its platforms. While these promises from the development team help encourage people to join, they also leave Pillowfort with extremely limited options for generating revenue. Furthermore, Pillowfort's commitment to allowing NSFW content on their site caused PayPal to sever ties with them, meaning that staff have had to turn to less well-known payment transaction services that require credit or debit cards. This restriction limited the different ways users could donate to Pillowfort.

U8: Can I donate to Pillowfort using Paypal? I don't have a credit card.

U9: Not being able to use PayPal is an issue for us Europeans. Not a lot of us have credit cards and I can only pay for things internationally with PayPal.

Pillowfort's identity is dependent upon allowing mature and NSFW content that other social media platforms don't allow. That same content is also not well-suited for sites that host only sexually explicit content. Pillowfort designs itself to be an in-between space, where fan artists and other creators can share a wide range of content that fits across multiple messy categories. Because Pillowfort's identity is grounded in being non-commercial as well as in support of NSFW content, the platform has relatively few paths to financial stability in the long term. Pillowfort's model does not fit well with how most social media sites function, meaning that it has less access to resources that are available to mainstream, commercialized social media platforms [7, 44].

It is these very commitments that position Pillowfort as different from mainstream social media platforms like Facebook and Twitter. Despite users joining Pillowfort and staying because it is distinctly not a major commercial social media platform, many still had the expectations that 
Pillowfort could operate like a major investment-funded platform. Specifically, users continually brought up that Pillowfort should begin hosting video content to compete with YouTube:

U10: I just submitted the feedback survey, but I want to emphasize that video hosting would be wonderful, considering that YouTube has been going downhill fast these days.

U11: Pillowfort is an overdue breath of fresh air, but the site can be even better! Let it strike while the iron is hot and implement a video-making system. YouTube's going the way of Photobucket. Pillowfort could overthrow them in the future!

Pillowfort does not have video hosting services. Instead, people can embed links to videos hosted elsewhere. Because Pillowfort is primarily user-funded, video hosting is most likely not an option within the platform's budget, at least not right now, representing a social-technical gap in Pillowfort's capabilities [1]. When people asked about video hosting options, staff tended to ignore the questions entirely. Video hosting is also not included in any of Pillowfort's official roadmaps or suggested improvements in the near future.

As far as funding Pillowfort goes, some users pay a registration fee while others set up regularly occurring donations. Users who have already joined can distribute three free registration keys to people who cannot pay or to friends. While putting a paywall on membership limits how fast Pillowfort can grow, it also helps to discourage people who would not fit with the community from joining. Pillowfort is leveraging intentional barriers to joining as a means of regulating its community, similar to how the site Metafilter used deliberate barriers to participation to ensure quality participation overall [54]. The site's slow growth model is most likely a result of working with a limited revenue stream, as Pillowfort most likely cannot support a large number of users who are unwilling to pay for its services.

Pillowfort draws in users willing to financially commit to the site by identifying itself as a safe space for fan communities to openly express themselves, particularly for fans that are part of the LGBTQ+ community and other marginalized groups like people of color. For example, in a post about the Black Lives Matter protests in 2020, Pillowfort staff stated their commitment to promoting diversity and inclusion on their platform and beyond. Pillowfort staff also declared that while they had been intending to celebrate Pride Month in 2020, they would be scaling back plans to hold space for the Black Lives Matter protests. Staff encouraged users to provide links to resources, charities, and funds that support Black Lives Matter and the LGBTQ+ community.

Staff: We offer solidarity to people fighting to be heard. We created Pillowfort to be an inclusive and safe space for diverse communities. Some users have asked for ways to support our fellow community members. We want to reach out and ask our community for a better answer to this question. Do you know about different charities or funds supporting these groups? Comment in the thread so people can find them.

The staff's efforts to cultivate this particular identity for Pillowfort mostly works. However, while Pillowfort's users see it as a safe space for now, they fear that toxic fan communities might eventually migrate to the site. Specifically, users express concerns about Pillowfort being a new home for anti-fan groups (e.g. fans whose activities center on expressing dislike or hatred toward a media property; [16]) because of a policy change the site made in December, 2018. Partly in response to an influx of artists fleeing from Tumblr, Pillowfort changed their NSFW content policy to ban sexually explicit visual artwork of underaged fictional characters. To be clear, users were not upset that explicit images of fictional minors were banned from the site. However, users were concerned that this ban could be leveraged by trolls and anti-fans to police fanworks. As an example, some users were concerned that people would report mature artwork of fictional characters that were canonically in their teens but had been illustrated as adults. 
U12: So Pillowfort says they won't look up a character's canonical age, but what if some troll sends them fanart of two characters engaged in sexually explicit activities and tells them, "These are underage characters," then is Pillowfort going to delete it?

Other social media platforms like Tumblr and LiveJournal implemented strict content bans, meaning that Pillowfort's policy revision, while not necessarily restrictive, made users question if Pillowfort would become unsafe to share mature content like other platforms had. Similar content bans on LiveJournal led to site-wide deletions of mature LGBTQ+ fan art, and Tumblr's adult content ban hurt marginalized groups like trans communities more than others [34]. Previously, Pillowfort's policy had simply stated that they would allow not-safe-for-work content to be hosted on their site but had not provided specific restrictions on what kinds of content would be acceptable or unacceptable.

Staff: We apologize for not clarifying our position sooner; we have been updating our terms of service to provide more clear guidelines. However, something happened today that made act with urgency, so made a statement as soon as possible...We know that this decision will anger and hurt some people, but we have spent a lot of time thinking about how to approach this and feel like we have made the best choice for our community.

Specifically, staff stated that they would not be extending bans to other "problematic" content that touched on difficult, traumatizing, or violent topics. The policy change was meant to provide clarity for how Pillowfort will be governed, but brought up concerns from users about bad-faith reporting and weaponized moderation tactics from anti-fan groups. As a specific example, many anti-fan groups have in the past bullied people on Tumblr for liking the "wrong" characters or would tell people certain characters are toxic or technically underage for certain periods in a story's canon [20]. One user afraid of this kind of behavior migrating to Pillowfort said:

U13: I can't be the only one thinking this will promote more anti-shipping behavior. We already had to go through this on Tumblr. There are endless block lists, harassment lists, and callout posts on Tumblr that specifically targets "grey area" material so that the people making that stuff would be endlessly harassed and then ultimately banned from the site.

The policy change highlights how Pillowfort must brand itself not only as a safe space for fan communities, but also a space safe from toxic and bullying behaviors found elsewhere on the internet. Public shaming and bullying tactics like U8 describes are something users encounter both outside of and within their own communities, supposed "safe spaces" [20]. The policy change also reminded users of other platforms they were fleeing when they joined Pillowfort, a move that potentially breaks the trust that Pillowfort's team worked to foster with users.

\subsection{Trust in Pillowfort}

As a user-funded platform, Pillowfort's staff is highly motivated to maintain trust with their users. Pillowfort staff engaged with their users extensively, making use of regular community surveys in addition to the public discussion posts where staff would respond individually to user comments and questions. Their regular public interactions also help users remain updated on recently implemented changes and proposed changes to the site. To foster this trust, the staff would directly ask for feedback on recent changes or proposed changes, whether through a community survey or in discussion threads themselves.

Staff: Using your survey feedback, we've been developing improvements to Communities, and we want to share what those improvements will look like with everyone. 
We want to hear what you think about these possible changes! It's important that we make sure these improvements will be something you folks care about. Leave your thoughts in the comment section. We'll be reading through your responses.

The staff's outreach efforts do not go unnoticed or unappreciated either. Users acknowledge that their replies to these staff posts are being read and, more importantly, their concerns are being listened to. Users often expressed their gratitude to the staff in follow-up comments on many of the threads, with the most common replies to any staff post being along the lines of, "thank you for all that you do!" Even when a site update was not to a user's liking, regular users still acknowledged the work that goes into maintaining Pillowfort.

U14: Thank you! I appreciate you guys and all that you do for us. My only complaint is that this update makes it harder for me to use the site until there's more user controls for this feature (and this is the only update I've disliked so far). I may keep talking about it in the thread, but I appreciate knowing that staff are listening.

U15: The rest of this update seems really great! Pillowfort keeps getting better and I trust you guys to keep it on the right track.

However, the trust that Pillowfort's staff works to foster with users can just as easily be broken. For example, users lost a significant amount of trust in the site when Pillowfort implemented the December 2018 policy change on explicit content. In contrast to other changes to the site, the policy change was not discussed or shared with the community before it was implemented. Rather, it was announced in response to a sudden influx of users from Tumblr. The update to the platform's policy and terms of service was announced without soliciting feedback from the community.

Users felt like they had lost trust in the platform, not necessarily because users desperately wanted to be able to draw and share dubious content, but because policies similar to the one written by Pillowfort had been used to remove content that other platforms found morally objectionable, such as sexually explicit content about LGBTQ+ people.

U16: I am thinking about leaving Pillowfort for somewhere else because I can't trust the staff not to ban things arbitrarily. This policy change will only hurt the community! Those 'grey areas' can easily backfire and be manipulated for abuse.

U17: I don't feel like this will be a platform I can trust anymore. I was hoping Pillowfort would be a place like Archive of Our Own where we'd stop fighting over gray areas and just hold each other accountable for managing our own experiences. Now I have doubts and it's disheartening.

U18: I can't really trust this policy in the current fandom climate. We don't know how this moral judgement and moderating this new rule might shift over time as Pillowfort grows. It feels like a time bomb, waiting to go off.

While Pillowfort's developers regularly will seek feedback from the community about design changes before implementing them, it does not consult the community on major policy changes before implementing them. Instead, Pillowfort developers will post an announcement explaining a recent change and why it took place, usually with an offer to help people process refunds if they no longer wish to support Pillowfort. In the following subsection, we discuss the differences in how Pillowfort engages with policy change announcements versus negotiating design implementations.

4.3.1 Feedback on Policy versus Design. In cases where Pillowfort implemented policy changes, they did not solicit feedback from users. This is most likely because policy must answer to constituents that are not Pillowfort's users first. For example, Pillowfort cannot host any content that violates U.S. law and must maintain a certain standard of practice that ensures other services will continue to work with them [38]. However, in a November 2019 policy change (when Pillowfort restricted 
viewable content for underage accounts) staff also announced a suite of design changes they had been proposing to users leading up to the policy change announcement.

In the December 2018 staff post announcing the updated NSFW policy, there were long threads of users expressing their frustration about the policy change and why it was a bad idea to make such a change. Users argued with one another as well as with staff over what the correct path forward for the site should be. Discourse between users quickly became divisive, but staff did not intervene to reply nearly as often as they did in the November 2019 update. In the December 2018 update, users expressed concern over everyone arguing:

U19: Wherever this update leads, can we just keep Pillowfort a place where we disagree with one another with civility instead of text-screaming and cursing at the small admin team that had no way of knowing this site would take off so quickly? I mean, it's nice when they take a moment to respond to our concerns. But we also need to accept that they're just starting out, and mistakes will be made.

In contrast to the December 2018 update, users were more eager to engage with staff during the November 2019 announcement with suggestions for design changes, and staff responded to feedback. This difference in response may come from the nature of the December 2018 policy change. First, its announcement came close on the heels of Tumblr's announcement of adult content being banned. Second, the November 2019 announcement focused on providing stronger filtering and control over who viewed what kind of content. It might also be possible that the number of users under 18 on Pillowfort is a negligent amount of the population, thus very few accounts were actually affected by the policy change. The following is one of the exchanges between staff and users in response to the November 2019 update:

U20: I'm left wondering some important things, such as how will this update affect how people view sex education and medical-related things? It's not easy to gauge stuff like that, but does the staff have guidelines in place for tagging stuff that's not porn but still sex-related?

Staff: This is a really good question and something our team wants to think about. Thank you for bringing this up. We're going to work on refining our guidelines to have something for users to consult.

Policy is clearly something that Pillowfort users are passionate about, and because of this, changes in policy can create tension between staff and users when that policy reminds them of past mistakes on other platforms. The policy change in December of 2018 made users feel uncomfortable because it was announced after the staff had taken moderation steps and removed a user's content that would violate the new policy. Though the action was taken to preserve Pillowfort as a safe and welcoming space, the way it was executed (content removal first, policy announcement later) was too similar to how other platforms mismanaged content.

Our findings demonstrate a balancing act that Pillowfort staff must maintain in developing the site, building out new features, and responding to user feedback in an engaging way. Furthermore, Pillowfort must do all of this without the resources available to major social media platforms and without receiving funding from sources that would compromise Pillowfort in the eyes of its users. In considering Pillowfort's struggles, we focus on three major points of discussion: (1) how the success and failure of new social media platforms are impacted by political tensions, (2) how those political tensions dictate design and policy decisions for social media platforms, and (3) considerations for designing and governing alternative social media platforms like Pillowfort. 


\section{DISCUSSION}

These findings point to certain design features and policy decisions that Pillowfort staff leveraged as core values to draw users in and keep them engaged. However we also see that there are certain choices the developers commit to that present challenges to maintaining the site. For example, the decision to host NSFW content cut off Pillowfort from using PayPal, limiting their payment processing options for users seeking to subscribe or donate to the site's maintenance costs. Paired with Pillowfort's mission statement, which emphasizes that the platform refuses to turn to investment or venture capital funding, the site is left with a significant challenge in that it has very few ways to generate a revenue capable of supporting Pillowfort and its developers Pillowfort's values as a platform and community are at odds with the site's ability to pay for server space and developer wages.

Throughout our findings, there were instances like PayPal severing ties where Pillowfort's developers and users shared a certain value, but those values were put at odds by more powerful systems. That is, Pillowfort's developers and users broadly wanted similar things. Staff communicated regularly with their users to elicit values that should be incorporated into the platform's ongoing development [30]. However, there are moments where users wanted Pillowfort to offer things that it physically could not, like video hosting services [1] or a guarantee that policy changes would not adversely affect NSFW content they wanted to share [38]. These sorts of issues do not necessarily represent tensions between competing values, but instead point to areas where values are being overridden by broader political tensions that Pillowfort's team must negotiate.

In considering what this case study illustrates, we explore three important concepts: (1) barriers to success and pathways to failure in starting new social media platforms; (2) how values in designing social media platforms intersect with politics in increasingly important ways; and (3) strategies we might consider in designing and governing alternative social media platforms.

\subsection{Success and Failure Revisited}

Even in beta, Pillowfort had to contend with significant challenges to success. The developers had limited access to funding, relying on an initial Kickstarter and user subscriptions, purposefully refusing to generate income through traditional methods. Pillowfort also must deal with its target audience comparing the platform's design and policy decisions to other platforms like Tumblr. Users were quick to mistrust Pillowfort's intentions when the site made policy decisions that reminded users of unfriendly policies on past platforms like LiveJournal and Tumblr. Users were not upset at the decision itself, but what the decision (and the action of deleting content) represented on other social media platforms in the past and what it could represent for Pillowfort's future.

In the past, CSCW scholars have framed an online community's success and failure around encouraging commitment from enough users to join the platform so that it reaches critical mass $[14,41]$. Oliver, Marwell, and Teixeira defined reaching critical mass as, "a small segment of the population that chooses to make big contributions to the collective action" (p. 524 [53]). In the context of Pillowfort, this definition still holds truth. The platform has had opportunities to rapidly grow its user base, especially in 2018 when users were fleeing Tumblr. However, Pillowfort not only remained in a closed beta then, it also introduced an update to their terms of service that was unpopular with current and incoming users, limiting the amount of people who would ultimately commit to the platform. When Pillowfort finally launched into an open beta in January, 2021, it quickly went offline and stayed offline for several months while the developers addressed several security risks.

These actions suggest that Pillowfort is, at the moment, not interested in becoming the next major social media platform. Instead, Pillowfort's staff are seeking success through reaching that 
critical mass of a small segment of dedicated users. The people that Pillowfort is encouraging commitment from are a niche audience: artists and fan communities interested in sharing art and discussing fandom-specific topics in small community spaces. Their users are also concerned with privacy and control over their content more than the average person on the internet $[11,19]$. Their users are interested in being able to post and share mature content that is not well-suited for platforms like Facebook, but also would not fit on a platform like Pornhub. In short, Pillowfort has worked hard to attract people who wish to use the platform for its intended purpose [33].

Whether or not Pillowfort's staff hope to remain a platform for niche interest groups, there are also major barriers to Pillowfort's continued success that influence the kinds of resources Pillowfort has access to. The platform's commitment to hosting mature content cost the developers their relationship with PayPal, thus limiting how easily they could collect revenue from a user-funded platform. Pillowfort does not host any content that is illegal under U.S. law (where the platform is based). However, PayPal made a judgement to sever ties with Pillowfort based on its own policies that are tied up in broader political issues. The decision is reflective of certain values that PayPal as a company projects, but is by no means an evenly applied rule. For example, PayPal uses a subsidiary to process payments to certain Patreons that host mature or "adult" content [4].

As a small social media platform, Pillowfort does not have the same power to leverage deals or exceptions like Patreon does. It does not have the resources to shift the broader politics affecting the internet in its favor, and because it does not have those resources, Pillowfort will continue to lack them. As Jessa Lingel puts it, "[C]ompeting with new platforms like Facebook and Instagram becomes a losing battle as they are out-spent and out-coded by the seemingly endless resources of Big Tech" (p. 11-12 [44]). The current political landscape of the internet favors large platforms that profit off of multitudinous commodification practices [7]. Pillowfort will not engage in many of those practices, and so it has more barriers to success to surmount before it escapes failure. However, the same decision to not engage in those practices is also a political decision on behalf of Pillowfort's developers, one that shifts power and some measure of control back into their hands.

\subsection{The Politics of Designing Platforms}

Above all else, the conversations between Pillowfort staff and their users highlights a necessary amount of politics that go into negotiating the platform's design and policy. More importantly, those politics matter just as much to the people using Pillowfort as they do to the staff. In recent years, it has become easy to imagine that these politics stop mattering once a social media platform reaches a certain scale, with Facebook somehow remaining unwavering in its popularity despite scandals that would normally have the potential to end a social media platform $[8,35]$. It might be that Facebook is not as reliant on its users for success anymore. Rather, the company's success is determined by how useful it can be to investors and other entities paying for one of the many different services the platform can offer.

In contrast, Pillowfort is beholden to its users by the site's own design and policy decisions. As an entirely user-funded platform, whether through donations or subscriptions, Pillowfort is a service to the people and their communities that use the platform. People come to Pillowfort because they do not want personalized advertisements, nor do they want algorithmic content matching, and neither do they want the problems they have encountered on sites like Tumblr or Twitter. They join because they want a site free from some of the more restrictive policy decisions on other platforms. Pillowfort's developers can say they will allow users to share NSFW content, but they can only make that promise insofar as they make sure to comply with the laws and regulations that permit them as a platform to continue operating [38].

The political values embedded in Pillowfort are displayed on the site's homepage: content control and privacy, user-created communities, blacklisting, blocking, threaded comments, and NSFW 
content allowed. Each of these features are positioned in contrast to how major social media platforms operate, and they communicate certain values that invite people to join who are looking for those political commitments. Langdon Winner states,

It is obvious that technologies can be used in ways that enhance the power, authority, and privilege of some over others...To our accustomed way of thinking, technologies are seen as neutral tools that can be used well or poorly, for good, evil, or something in between. But we usually do not stop to inquire whether a given device might have been designed and built in such a way that it produces a set of consequences logically and temporally prior to any of its professed uses. (p. 125 [61])

Just over fourty years after Winner wrote this, we are still perhaps too hesitant to view technologies as being built with a certain set of politics that govern their use. Pillowfort surfaces its politics by projecting values toward privacy and user-centered control over an online experience. In doing so, Pillowfort is not positioning itself as morally superior or better than other social media platforms, but it is advertising a specific kind of experience that users cannot find elsewhere online. Platforms like Facebook can offer features like user-generated communities and granular privacy controls, but the underlying politics of Facebook-its profit model, its focus on generating engagement, algorithmically-managed communities and news feeds-positions Facebook as an antithetical experience to what people joining Pillowfort are looking for.

Pillowfort made certain promises to its users-that it would put user privacy first and avoid certain types of revenue. These values allow Pillowfort to maintain a certain amount of control and power in its own development, though they still come with their own trade-offs. Taken together, their values form a political gesture, much like Pillowfort's policy decisions toward which kinds of explicit artwork they will and will not host. Decisions on what kind of content to host, how to facilitate interaction between users, and how to enforce the policies that govern those users are all situated within the politics of a platform's design.

\subsection{Considerations for Designing \& Supporting Alternative Platforms}

Pillowfort is not the first social media platform to emerge as a countercultural space to oppose larger platforms. The Body Modification Ezine, for example, emerged as digital counterculture in the early years of the internet and eventually struggled to compete with large social media platforms like Facebook [43]. When fan communities fled LiveJournal, platforms like Archive of Our Own (AO3) and Dreamwidth formed as alternatives [22]. Existing within the non-profit Organization for Transformative Works (OTW), AO3 is uniquely positioned to offer certain things that other sites cannot-a safe space to host fanworks of any variety, regardless of a single person's moral values projected onto those fanworks [23]. In addition to maintaining AO3, the OTW conducts legal advocacy work to support the safe existence of fanworks [29].

The OTW's advocacy work represents an instance of a community's values and everyday practices significantly transforming the politics around fanworks [46]. Like the OTW, Pillowfort has purposefully constructed a digital platform divested from specific funding sources and traditional social media practices. By doing so, Pillowfort might just affect broader political frameworks. Huybrechts and colleagues propose institutioning as a gradual process of altering, consolidating, and challenging existing institutions through participatory design practices [36]. Building on this definitional work, Teli and colleagues propose blending the concepts of "institutioning" and "commoning" in transformative design practices [58]. Their work proposes "commoning" as a participatory design practice that centers grassroots communities and movements, thus promoting change from outside an institution. 
In considering how we might further support and uplift grassroot communities like Pillowfort, we can start by examining the barriers they face in their own design practices and how they choose to navigate those barriers. In an investigation into supporting grassroot community radio groups, Cibin and colleagues identified several barriers local communities faced in procuring a radio license: complex bureaucratic processes, technical issues, and local politics to name a few [15]. Through leveraging local experts, those communities were able to succeed and enriched their own spaces in the process. In the case of Pillowfort, the community is scattered across the globe and the local experts available to staff are their own users. While those users often bring a wide range of expertise in computer programming issues, they are not always equipped to provide the exact expertise that Pillowfort's small staff might need in the moment. Pillowfort certainly demonstrates a willingness to source local talent from its users, a strategy that fan communities have leveraged historically $[6,23]$. Outside of fan communities, practitioners running digital platforms can leverage their own "local" experts in navigating design and resource challenges.

Pillowfort also is another example of how strong platform governance can be drawn from an online community's existing values [21]. By asking for feedback on design changes and engaging with the users directly over policy decisions, the developers draw values from the community to implement in upcoming changes. Even if Pillowfort ultimately does not integrate every recommended change from their users, the act of conversing with their userbase and explaining why certain design and policy decisions are made strengthens the community's sense of trust in Pillowfort's staff.

Keeping this issue in mind, we might consider future work that makes expertise available to small, countercultural digital platforms. Pillowfort does not have the same resources to leverage as its larger counterparts, so how do we make available the different experts in legal issues, cyber-security, and funding that more established tech companies have access to? Whether this take the shape of a volunteer collective or a resource library, there are multiple paths forward to providing this kind of support. We might also consider methods for supporting small platforms in acquiring and maintaining both the physical and digital infrastructure required to successfully operate a platform. Just like the Organization for Transformative Works advocates for and maintains Archive of Our Own, so too can advocacy groups support the development and success of those smaller, more grassroots social media platforms. In doing so, we might just gradually affect the current politics of designing platforms.

\section{CONCLUSION}

As social media platforms and their communities continue to evolve, platform developers will have to make increasingly challenging decisions about how a platform will engage with overarching political challenges. Users may never pack their bags and leave Facebook to the point where the site collapses, but they may take activities unwelcome on that platform elsewhere. The internet is a highly commercialized space, with power and resources quickly consolidating to a few key platforms. Something this case study offers is an observation of how different and new social media platforms are operating. It is easy to at first dismiss concerns about people taking their unwelcome activities elsewhere. Pushing extremist groups like anti-vaccine communities or militia groups onto resource-starved platforms makes them less effective than they would be on major social media platforms and it is better if the platforms they do use to organize remain small and struggling. However, there are still pockets of the internet that serve as a place of respite and creativity for marginalized groups like people of color and LGBTQ+ people, communities that might be pushed off major platforms unintentionally or perhaps even feel unsafe using those platforms. Issues that impact a small platform's ability to thrive will greatly impact these marginalized communities. 
The politics that most strongly influence social media platforms often crowd out more vulnerable groups, like we saw with Tumblr's adult content ban affecting trans communities [34]. While Pillowfort is a technology being developed specifically for marginalized communities, we see that its path to success is a narrow and difficult one. Despite those challenges, the site continues on, uncompromising thus far in its initial statement of values and goals. Moving forward, we hope to leverage this work to support and uplift marginalized people building and maintaining their own online communities and reduce the barriers they encounter in those pursuits.

\section{ACKNOWLEDGMENTS}

We would like to thank the members of the Internet Rules Lab (IRL) for their support throughout the research process. In particular, we would like to thank Blakeley Payne and Ellen Simpson for their close readings of early drafts of the article. This work was funded by NSF award 1936741.

\section{REFERENCES}

[1] Mark S Ackerman. 2000. The intellectual challenge of CSCW: The gap between social requirements and technical feasibility. Human-Computer Interaction 15, 2-3 (2000), 179-203.

[2] Julia Alexander. 2018. Pillowfort wants to be the Tumblr alternative, but it's not ready yet. The Verge (Dec 2018). https://www.theverge.com/2018/12/7/18127061/pillowfort-tumblr-exodus-dreamwidth-livejournal-adultcontent-december-17

[3] Muhammad Ali, Piotr Sapiezynski, Miranda Bogen, Aleksandra Korolova, Alan Mislove, and Aaron Rieke. 2019. Discrimination through optimization: How Facebook's Ad delivery can lead to biased outcomes. Proceedings of the ACM on Human-Computer Interaction 3, CSCW (2019), 1-30.

[4] Lux Alptraum. 2016. Patreon Ends Payments Discrimination Against Adult Content. Vice (Jul 2016). https://www. vice.com/en/article/gv5dmb/patreon-paypal-adult-content

[5] Judd Antin and Coye Cheshire. 2010. Readers Are Not Free-Riders: Reading as a Form of Participation on Wikipedia. In Proceedings of the 2010 ACM Conference on Computer Supported Cooperative Work (Savannah, Georgia, USA) (CSCW '10). Association for Computing Machinery, New York, NY, USA, 127-130. https://doi.org/10.1145/1718918.1718942

[6] Camille Bacon-Smith. 1992. Enterprising women: Television fandom and the creation of popular myth. University of Pennsylvania Press.

[7] Chiara Bassetti, Mariacristina Sciannamblo, Peter Lyle, Maurizio Teli, Stefano De Paoli, and Antonella De Angeli. 2019. Co-designing for common values: creating hybrid spaces to nurture autonomous cooperation. CoDesign 15, 3 (2019), $256-271$.

[8] Allison J Brown. 2020. "Should I Stay or Should I Leave?": Exploring (Dis) continued Facebook Use After the Cambridge Analytica Scandal. Social Media + Society 6, 1 (2020), 2056305120913884.

[9] Susan L. Bryant, Andrea Forte, and Amy Bruckman. 2005. Becoming Wikipedian: Transformation of Participation in a Collaborative Online Encyclopedia. In Proceedings of the 2005 International ACM SIGGROUP Conference on Supporting Group Work (Sanibel Island, Florida, USA) (GROUP '05). Association for Computing Machinery, New York, NY, USA, 1-10. https://doi.org/10.1145/1099203.1099205

[10] Julia Bullard. 2016. Motivating invisible contributions: framing volunteer classification design in a fanfiction repository. In Proceedings of the 19th International Conference on Supporting Group Work. 181-193.

[11] Kristina Busse and Karen Hellekson. 2012. Identity, Ethics, and Fan Privacy. Fan Culture: Theory/Practice (2012), 38-56.

[12] Julie Campbell, Cecilia Aragon, Katie Davis, Sarah Evans, Abigail Evans, and David Randall. 2016. Thousands of positive reviews: Distributed mentoring in online fan communities. In Proceedings of the 19th ACM Conference on Computer-Supported Cooperative Work \& Social Computing. 691-704.

[13] Eshwar Chandrasekharan, Umashanthi Pavalanathan, Anirudh Srinivasan, Adam Glynn, Jacob Eisenstein, and Eric Gilbert. 2017. You can't stay here: The efficacy of reddit's 2015 ban examined through hate speech. Proceedings of the ACM on Human-Computer Interaction 1, CSCW (2017), 1-22.

[14] Yao-Sheng Chang and Chyan Yang. 2013. Why do we blog? From the perspectives of technology acceptance and media choice factors. Behaviour \& Information Technology 32, 4 (2013), 371-386.

[15] Roberto Cibin, Maurizio Teli, and Sarah Robinson. 2019. Institutioning and community radio. A comparative perspective. In Proceedings of the 9th International Conference on Communities \& Technologies-Transforming Communities. 143-154.

[16] Melissa Click. 2019. Anti-fandom: Dislike and hate in the digital age. NYU Press.

[17] Paul Dourish. 2010. HCI and environmental sustainability: the politics of design and the design of politics. In Proceedings of the 8th ACM Conference on Designing Interactive Systems. 1-10. 
[18] Brianna Dym, Jed R Brubaker, Casey Fiesler, and Bryan Semaan. 2019. "Coming Out Okay": Community Narratives for LGBTQ Identity Recovery Work. Proceedings of the ACM on Human-Computer Interaction 3, CSCW (2019), 1-28.

[19] Brianna Dym and Casey Fiesler. 2020. Ethical and privacy considerations for research using online fandom data. Transformative Works and Cultures 33 (2020).

[20] Brianna Dym and Casey Fiesler. 2020. Social Norm Vulnerability and its Consequences for Privacy and Safety in an Online Community. Proceedings of the ACM on Human-Computer Interaction 4, CSCW2 (2020), 1-24.

[21] Casey Fiesler and Amy S Bruckman. 2019. Creativity, Copyright, and Close-Knit Communities: A Case Study of Social Norm Formation and Enforcement. Proceedings of the ACM on Human-Computer Interaction 3, GROUP (2019), 1-24.

[22] Casey Fiesler and Brianna Dym. 2020. Moving Across Lands: Online Platform Migration in Fandom Communities. Proceedings of the ACM on Human-Computer Interaction 4, CSCW1 (2020), 1-25.

[23] Casey Fiesler, Shannon Morrison, and Amy S Bruckman. 2016. An archive of their own: A case study of feminist HCI and values in design. In Proceedings of the 2016 CHI Conference on Human Factors in Computing Systems. 2574-2585.

[24] Casey Fiesler, Shannon Morrison, R Benjamin Shapiro, and Amy S Bruckman. 2017. Growing their own: Legitimate peripheral participation for computational learning in an online fandom community. In Proceedings of the 2017 ACM conference on computer supported cooperative work and social computing. 1375-1386.

[25] Casey Fiesler and Nicholas Proferes. 2018. "Participant" perceptions of Twitter research ethics. Social Media + Society 4, 1 (2018), 2056305118763366.

[26] Mary Flanagan, Daniel C Howe, and Helen Nissenbaum. 2008. Embodying values in technology: Theory and practice. In Information Technology and Moral Philosophy, Jeroen Van Den Hoven and John Weckert (Eds.). Cambridge University Press, Chapter 16, 322-353.

[27] Diana Floegel. 2020. "Write the story you want to read": world-queering through slash fanfiction creation. fournal of Documentation (2020).

[28] Kerry Flynn. 2021. Facebook blocks news sharing in Australia in response to government proposal. CNN (Feb 2021). https://www.cnn.com/2021/02/17/media/facebook-australia-news-ban/index.html

[29] Organization for Transformative Works. [n.d.]. Legal Advocacy. https://www.transformativeworks.org/legal/

[30] Batya Friedman, Peter H Kahn, and Alan Borning. 2008. Value sensitive design and information systems. The handbook of information and computer ethics (2008), 69-101.

[31] James Paul Gee. 2014. How to do discourse analysis: A toolkit. Routledge.

[32] Sarah A Gilbert. 2020. "I run the world's largest historical outreach project and it's on a cesspool of a website." Moderating a Public Scholarship Site on Reddit: A Case Study of r/AskHistorians. Proceedings of the ACM on HumanComputer Interaction 4, CSCW1 (2020), 1-27.

[33] Jonathan Grudin. 1988. Why CSCW applications fail: problems in the design and evaluationof organizational interfaces. In Proceedings of the 1988 ACM conference on Computer-supported cooperative work. 85-93.

[34] Oliver L Haimson, Avery Dame-Griff, Elias Capello, and Zahari Richter. 2019. Tumblr was a trans technology: the meaning, importance, history, and future of trans technologies. Feminist Media Studies (2019), 1-17.

[35] Blake Hallinan, Jed R Brubaker, and Casey Fiesler. 2020. Unexpected expectations: Public reaction to the Facebook emotional contagion study. New Media \& Society 22, 6 (2020), 1076-1094.

[36] Liesbeth Huybrechts, Henric Benesch, and Jon Geib. 2017. Institutioning: Participatory design, co-design and the public realm. CoDesign 13, 3 (2017), 148-159.

[37] Mike Isaac and Kellen Browning. 2020. Fact-Checked on Facebook and Twitter, Conservatives Switch Their Apps. The New York Times (Nov 2020). https://www.nytimes.com/2020/11/11/technology/parler-rumble-newsmax.html

[38] Steven J Jackson, Tarleton Gillespie, and Sandy Payette. 2014. The policy knot: Re-integrating policy, practice and design in CSCW studies of social computing. In Proceedings of the 17th ACM conference on Computer supported cooperative work \& social computing. 588-602.

[39] Henry Jenkins. 1988. Star Trek rerun, reread, rewritten: Fan writing as textual poaching. Critical Studies in Media Communication 5, 2 (1988), 85-107.

[40] Henry Jenkins. 2012. Textual poachers: Television fans and participatory culture. Routledge.

[41] Robert E Kraut and Paul Resnick. 2012. Building successful online communities: Evidence-based social design. MIT Press.

[42] Christopher A Le Dantec and Carl DiSalvo. 2013. Infrastructuring and the formation of publics in participatory design. Social Studies of Science 43, 2 (2013), 241-264.

[43] Jessa Lingel. 2017. Digital countercultures and the struggle for community. MIT Press.

[44] Jessa Lingel. 2021. The Gentrification of the Internet: How to Reclaim Our Digital Freedom. University of California Press.

[45] Caitlin Lustig. 2019. Intersecting imaginaries: visions of decentralized autonomous systems. Proceedings of the ACM on Human-Computer Interaction 3, CSCW (2019), 1-27.

[46] Ezio Manzini. 2019. Politics of the Everyday. Bloomsbury Visual Arts. 
[47] Annette Markham. 2012. Fabrication as ethical practice: Qualitative inquiry in ambiguous internet contexts. Information, Communication \& Society 15, 3 (2012), 334-353.

[48] Adrienne Massanari. 2017. \#Gamergate and The Fappening: How Reddit's algorithm, governance, and culture support toxic technocultures. New Media \& Society 19, 3 (2017), 329-346.

[49] Adrienne L Massanari. 2019. "Come for the period comics. Stay for the cultural awareness": reclaiming the troll identity through feminist humor on Reddit's /r/TrollXChromosomes. Feminist Media Studies 19, 1 (2019), 19-37.

[50] Jessica K Miller, Batya Friedman, Gavin Jancke, and Brian Gill. 2007. Value tensions in design: the value sensitive design, development, and appropriation of a corporation's groupware system. In Proceedings of the 2007 international ACM conference on Supporting group work. 281-290.

[51] Chantal Mouffe. 2005. The Return of the Political. Verso.

[52] Safiya Umoja Noble. 2018. Algorithms of oppression: How search engines reinforce racism. NYU Press.

[53] Pamela Oliver, Gerald Marwell, and Ruy Teixeira. 1985. A theory of the critical mass. I. Interdependence, group heterogeneity, and the production of collective action. American journal of Sociology 91, 3 (1985), 522-556.

[54] Hannah Pileggi, Briana Morrison, and Amy Bruckman. 2014. Deliberate Barriers to User Participation on MetaFilter. Technical Report. Georgia Institute of Technology.

[55] Toni Robertson and Jesper Simonsen. 2012. Participatory Design: an introduction. In Routledge international handbook of participatory design. Routledge, Chapter 1,21-38.

[56] Nick Srnicek. 2016. Platform Capitalism. John Wiley \& Sons.

[57] Stefan Stieger, Christoph Burger, Manuel Bohn, and Martin Voracek. 2013. Who commits virtual identity suicide? Differences in privacy concerns, internet addiction, and personality between Facebook users and quitters. Cyberpsychology, Behavior, and Social Networking 16, 9 (2013), 629-634.

[58] Maurizio Teli, Marcus Foth, Mariacristina Sciannamblo, Irina Anastasiu, and Peter Lyle. 2020. Tales of institutioning and commoning: participatory design processes with a strategic and tactical perspective. In Proceedings of the 16th Participatory Design Conference 2020-Participation (s) Otherwise-Volume 1. 159-171.

[59] John Tulloch and Henry Jenkins. 1995. Science Fiction Audiences: Watching Doctor Who and Star Trek.

[60] Ana Valens. 2021. After security woes, Tumblr alternative Pillowfort promises relaunch. The Daily Dot (March 2021). https://www.dailydot.com/irl/pillowfort-april-relaunch/

[61] Langdon Winner. 1986. Do Artifacts Have Politics? In The Whale and the Reactor: A Search for Limits in an Age of High Technology. University of Chicago Press, Chapter 2, 19-39.

Received July 2021; revised September 2021; accepted October 2021 